Composite Structures

\title{
Stress distribution around an elliptic hole in a plate with 'implicit' and 'explicit' non-local models
}

\author{
--Manuscript Draft--
}

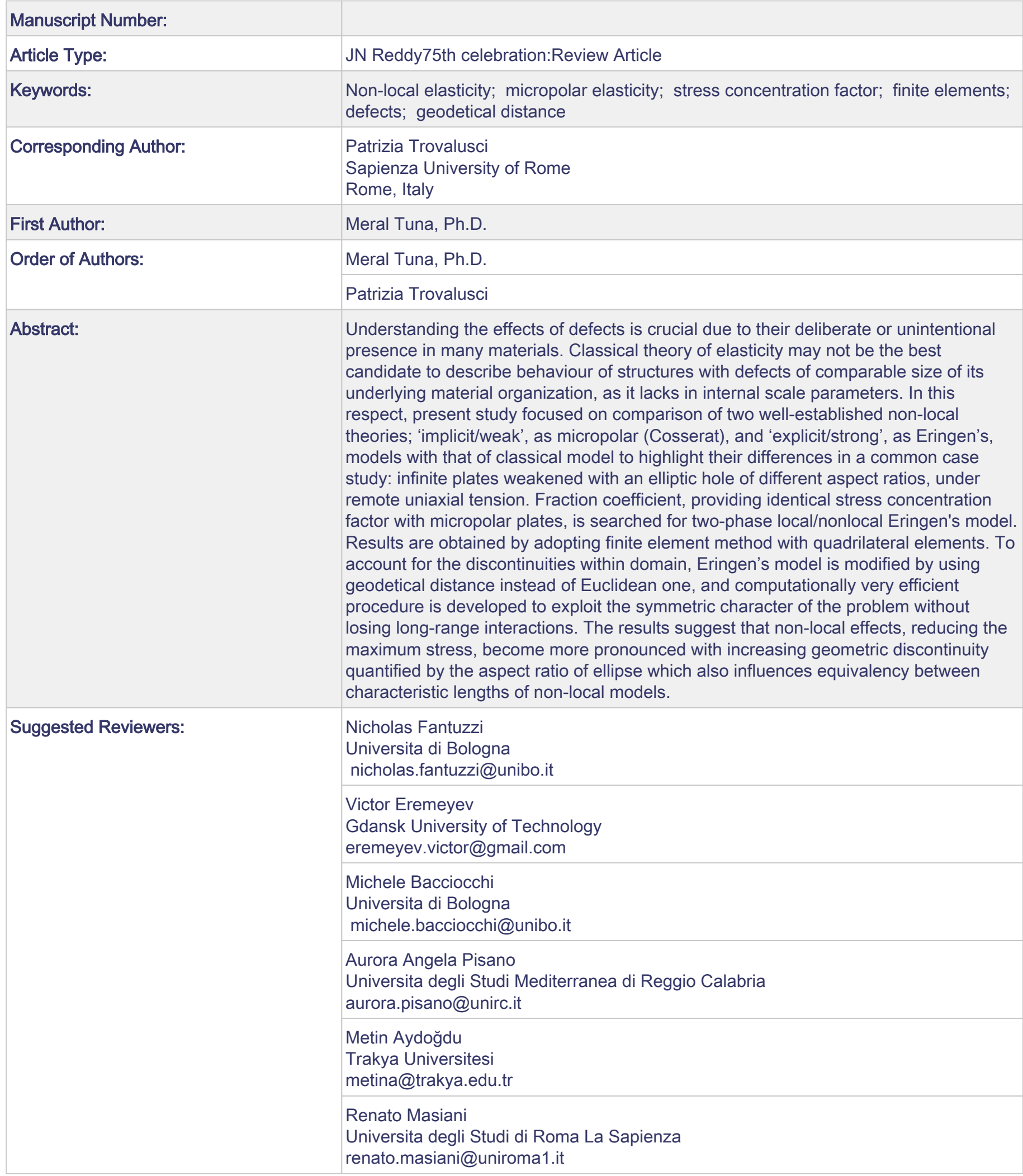




\section{Patrizia TROVALUSCI}

\section{SAPIENZA UNIVERSITY of ROME}

\section{Department of Structural and Geotechnical Engineering}

Via A. Gramsci,53, 00197 Rome - ITALY

E-mail : patrizia.trovalusci@uniroma1.it

Dear Professor Ferreira,

The manuscript entitled "Stress distribution around an elliptic hole in a plate with 'implicit' and 'explicit' non-local models", by Meral Tuna and myself, has been submitted online for possible publishing on Composite Structures. The paper contains novel contribution in the field of non classical, size dependent, continuum formulations for heterogeneous materials.

Sincerely Yours, Rome, July 15th, 2020

Patrizia Trovalusci, Ph.D.

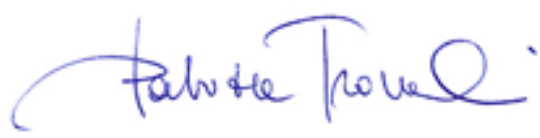




\section{Highlights}

- Stress fields of non-local models are compared with classical model for a case study.

- Maximum stress decreases with increased non-locality, especially for sharper holes.

- An equivalency between characteristic lengths of non-local models is attained.

- Eringen's formulation is modified by adopting geodetical distance instead of Euclidean.

- A validated symmetric finite element model is proposed for Eringen's model. 


\title{
Stress distribution around an elliptic hole in a plate with 'implicit' and 'explicit' non-local models
}

\author{
Meral Tuna ${ }^{\mathrm{a}}$, Patrizia Trovalusci ${ }^{\mathrm{b}, *}$ \\ ${ }^{a}$ Istanbul Technical University, Faculty of Mechanical Engineering, Inonu Caddesi, 65, 34437 Istanbul, Turkey \\ ${ }^{b}$ Department of Structural and Geotechnical Engineering, Sapienza University of Rome, Via A. Gramsci, 53, 00197, Rome, Italy
}

\begin{abstract}
Understanding the effects of defects is crucial due to their deliberate or unintentional presence in many materials. Classical theory of elasticity may not be the best candidate to describe behaviour of structures with defects of comparable size of its underlying material organization, as it lacks in internal scale parameters. In this respect, present study focused on comparison of two well-established non-local theories; 'implicit/weak', as micropolar (Cosserat), and 'explicit/strong', as Eringen's, models with that of classical model to highlight their differences in a common case study: infinite plates weakened with an elliptic hole of different aspect ratios, under remote uniaxial tension. Fraction coefficient, providing identical stress concentration factor with micropolar plates, is searched for two-phase local/nonlocal Eringen's model. Results are obtained by adopting finite element method with quadrilateral elements. To account for the discontinuities within domain, Eringen's model is modified by using geodetical distance instead of Euclidean one, and computationally very efficient procedure is developed to exploit the symmetric character of the problem without losing long-range interactions. The results suggest that non-local effects, reducing the maximum stress, become more pronounced with increasing geometric discontinuity quantified by the aspect ratio of ellipse which also influences equivalency between characteristic lengths of non-local models.
\end{abstract}

Key words: Non-local elasticity, micropolar elasticity, stress concentration factor, finite elements, defects, geodetical distance

\section{Introduction}

The studies of Voigt $[1,2]$ and Poincaré $[3]^{1}$ can be considered as the foundation of what we call today as nonlocal media, which, in general, is characterized by the presence of internal length parameters and spatial dispersion properties [8-10].

It has been now well-acknowledged that resorting to non-classical continuum formulations for representing the behaviour of materials with internal structure (composites, nanomaterials, biomaterials, etc.) is actually very tempting due to their ability on maintaining the information of underlying material organization, with particular reference to material's internal lengths, yet utilizing the advantage of field description at coarse level [11-16]. Thereby, many non-classical theories, with scale parameters referring to different physical features from nanoorders (e.g. distance between atoms in a graphene sheet) to meso/macro-orders (e.g. size of a particle in a composite medium or size of a block in masonry wall) have been proposed, while a systematic treatise can be achieved by

\footnotetext{
${ }^{*}$ Corresponding author

Email addresses: tunamer@itu.edu.tr (Meral Tuna), patrizia.trovalusci@uniroma1.it (Patrizia Trovalusci)

${ }^{1}$ The readers refer to [4], [5], [6], [7] for a comprehensive survey on discrete to continuum approaches.
}

following the classification of [12], [14], [15], adopted in [17], as 'weak/implicit' and 'strong/explicit'. As the focus of the present study is limited to implicit-type micropolar (Cosserat) and explicit-type Eringen's non-local models, the mentioned categorization is explained on the basis of these two.

Micropolar theory belongs to a group of generalised continua with additional degrees of freedom (DOFs). Here, the material body is considered as a collection of rigid particles that are enriched with rotational DOFs, and interact through not only forces but also couples [15, 1821]. Since non-locality is introduced to the model solely through additional (non-standard) kinematic and their work-conjugated dynamic descriptors, the theory maintains a weak non-local character, and appears as the best fit for describing the materials with presence of particle rotations (e.g. heterogeneous materials with inclusions/voids such as; fiber-reinforced composites, and heterogeneous materials with microstructure such as; cellular material, jointed rock, masonry) [22-35]. On the other side, as one of the most resorted 'explicit' type non-local theory, Eringen's non-local model interests with material bodies in which all points interact with each other depending inversely on the distance in-between $[36,37]$. The theory covers the long-range interactions through an attenuation type kernel function that is directly included in 
the constitutive equation, hence yielding a strong non-local character. Wherefore, Eringen's theory -with its enhanced versions - seems to be mainly conducted in investigation of structures exhibiting neighbouring attractions (e.g. nano or micro sized materials such as; molecular arrays, carbon nanotubes, atomic-sized sensors) [38-50].

Possessing additional kinematic and dynamic descriptors significantly enriches the description of the non-local theories, while in order to better understand their capabilities, limitations and similarities, many comparative studies have been conducted [51-56]. With a similar motivation, the focus here is on plates having either -easier to handlecircular hole or -more generic- elliptic hole. Literature survey shows that; besides a recent paper of the authors [56], the considered problem has been investigated only on the basis of micropolar theory looking for numerical or exact solutions $[15,57-63]$.

In order to compare these two non-local solutions to that of classical one, stress fields of infinite plates weakened with a central elliptic hole of different aspect ratios, subjected to remote uniaxial tension are examined in terms of local Cauchy, non-local 'implicit', as micropolar (Cosserat), and non-local 'explicit', as Eringen's theories. The numerical solutions are obtained by employing finite element (FE) method within linear elastic framework and considering isotropy. The domain is discretised with a generic mesh configuration consisting of four-node linear quadrilateral elements. Stress concentration factors (SCF) of infinite Cauchy and micropolar (Cosserat) plates are compared with analytical results reported in literature $[57,64]$ for validation of the FE models. To increase the computational efficiency, all the simulations are performed using symmetric models. Although such rearrangement in the FE models of Cauchy and micropolar (Cosserat) plates are straightforward (i.e. considering only quarter portion of the domain with imposing symmetry related boundary conditions is sufficient), a new approach that conveys the necessary long range effects, yet reduces total degrees of freedom, is introduced for Eringen's model. The concept can be regarded as a generalisation of what has been proposed by [65], while here we exploit direct evaluation method instead of an iterative approach. As the existence of geometric discontinuities within the domain necessitates the incorporation of geodetical path for correct evaluation of the long range interactions [66], a robust and effective strategy is presented to customise the Eringen's formulation. As the last step, the non-locality of two phase local/nonlocal Eringen's model is tuned through fraction coefficient to have stress concentration factors in accordance with micropolar plates. The selection of fraction coefficient to this aim is justified with the consideration of computational burden brought by the requirement to alter the influence zone and to calculate the stiffness matrices with changing non-local parameter.

\section{Materials and methods}

\subsection{Overview}

This section provides general information on micropolar theory and integral form of two phase local/nonlocal Eringen's theory, presenting their limit cases leading to Cauchy continua. Corresponding two-dimensional (2D) displacement-based $\mathrm{FE}$ formulations are presented for a generic non-uniform spatial discretisation using quadrilateral elements under the assumption of linear elasticity and plane-strain. The structure under investigation is a plate of uniform thickness, $h$, with an elliptic void, and is made of linear and isotropic material. Regarding Eringen's theory, a special attention has been paid to boundary conditions which enables to exploit symmetry for FE model along with the incorporation of geodetical distance. A Cartesian coordinate system is used for parametrization with $z$ axis along the thickness. The superscripts $M$ and $E$, refer to micropolar (Cosserat) and Eringen's non-local models respectively, used to distinguish the parameters emerged in both. The FE formulations are implemented to an in-house Mathematica code for performing the simulations.

\subsubsection{Micropolar (Cosserat) model}

In the so-called implicitly non-local micropolar theory, the material particles can be conceived as a collection of rigid bodies, which undergo both translations and rotations. Hence, in a 2D framework, out of plane microrotation component $\left(\phi_{z}\right)$ is added to the classical in-plane displacement components $\left(u_{x}, u_{y}\right)$ to define the motion of the body. Let us consider a linearised theory; the strain measures are represented by the strain $\left(\varepsilon_{i j}^{M}\right)$ and curvature $\left(\chi_{k j}\right)$ tensor. The kinematic compatibility equations write:

$$
\varepsilon_{i j}^{M}=u_{i, j}+e_{i j k} \phi_{k}, \quad \chi_{k j}=\phi_{k, j}
$$

where $e_{i j k}$ is permutation symbol. Unlike the classical theory of elasticity, the presence of relative rotation (i.e. the difference between micro and macro rotations);

$$
\phi_{k}-\frac{1}{2} e_{k m n} u_{n, m} \neq 0
$$

spoils the symmetry of strain tensor, which can be recovered only if micro rotations $\left(\phi_{k}\right)$ are forced to follow the local rigid rotation (macrorotation: $\frac{1}{2} e_{k m n} u_{n, m}$ ), yielding a couple stress continua [22, 67-69].

In the absence of body forces and couples, conservation of linear and angular momentums, along with Green's theorem, provides the following bulk balance equations:

$$
\sigma_{i j, j}^{M}=0, \quad \mu_{k j, j}-e_{i j k} \sigma_{i j}^{M}=0
$$

with the surface balance equations:

$$
t_{i}^{M}=\sigma_{i j}^{M} n_{j}, \quad m_{k}=\mu_{k j} n_{j}
$$

Here $\sigma_{i j}^{M}$ and $\mu_{k j}$ refer to non-symmetric and couple stress tensors respectively, while $t_{i}^{M}$ and $m_{k}$ denote traction and 
couple traction. $n_{j}$ is the unit outward normal vector to the boundary.

In the context of linear elasticity and isotropy, the constitutive relations between strain/curvature and the workconjugated stress/couple-stress tensors are expressed in the following form:

$$
\begin{aligned}
\sigma_{i j}^{M} & =\lambda \varepsilon_{k k}^{M} \delta_{i j}+(\mu+\chi) \varepsilon_{i j}^{M}+\mu \varepsilon_{j i}^{M}, \\
\mu_{k j} & =\alpha \chi_{i i} \delta_{k j}+\beta \chi_{j k}+\gamma \chi_{k j}
\end{aligned}
$$

where $\lambda$ and $\mu$ refer to generalised Lamé constants:

$$
\lambda=\frac{E \nu}{(1+\nu)(1-2 \nu)}, \quad \mu=G-\frac{\chi}{2}
$$

Then the Poisson's ratio, $\nu$, is expressed as

$$
\nu=\frac{\lambda}{2(\lambda+G)}=\frac{\lambda}{2 \lambda+2 \mu+\chi}
$$

with $\alpha, \beta, \gamma$ and $\chi$ being constants related to micropolar theory.

Eq. (5) can be reorganized to a matrix form as

$$
\left\{\begin{array}{l}
\boldsymbol{\sigma}^{M} \\
\boldsymbol{\mu}
\end{array}\right\}=\left[\begin{array}{ll}
\mathbf{D}_{\varepsilon}^{M} & \mathbf{0}_{4 \times 2} \\
\mathbf{0}_{2 \times 4} & \mathbf{D}_{\chi}
\end{array}\right]\left\{\begin{array}{l}
\varepsilon^{M} \\
\chi
\end{array}\right\}
$$

where strain, curvature, stress and couple-stress components are respectively ordered in the following vectors:

$$
\begin{array}{llll}
\boldsymbol{\sigma}^{M}=\left\{\begin{array}{lllll}
\sigma_{11}^{M} & \sigma_{22}^{M} & \sigma_{12}^{M} & \sigma_{21}^{M}
\end{array}\right\}^{T}, & \boldsymbol{\mu}=\left\{\begin{array}{lll}
\mu_{31} & \mu_{32}
\end{array}\right\}^{T} \\
\boldsymbol{\varepsilon}^{M}=\left\{\begin{array}{lllll}
\varepsilon_{11}^{M} & \varepsilon_{22}^{M} & \varepsilon_{12}^{M} & \varepsilon_{21}^{M}
\end{array}\right\}^{T}, \quad \boldsymbol{\chi}=\left\{\begin{array}{lll}
\chi_{31} & \chi_{32}
\end{array}\right\}^{T}
\end{array}
$$

For plane-strain, elasticity matrices take the following forms:

$$
\begin{aligned}
& \mathbf{D}_{\varepsilon}^{M}=\left[\begin{array}{cccc}
\lambda+2 G & \lambda & 0 & 0 \\
\lambda & \lambda+2 G & 0 & 0 \\
0 & 0 & G+\frac{\chi}{2} & G-\frac{\chi}{2} \\
0 & 0 & G-\frac{\chi}{2} & G+\frac{\chi}{2}
\end{array}\right] \\
& \mathbf{D}_{\chi}=\left[\begin{array}{ll}
\gamma & 0 \\
0 & \gamma
\end{array}\right]
\end{aligned}
$$

The theory incorporates size effects and relative rotations through internal characteristic length, $l_{c}$, and coupling number, $N[70]$.

$$
l_{c}^{2}=\frac{\gamma}{2(2 \mu+\chi)}, \quad N^{2}=\frac{\chi}{2(\mu+\chi)}
$$

In the light of Eqs. (10) and (11), it is fair to interpret that the bending moduli $\mathbf{D}_{\chi}$ is responsible for scale effects.

In the case in which $l_{c}$ and $N$ are small enough, for a material that belongs at least to orthotetragonal symmetry class as the isotropic one, the micropolar model reduces to Cauchy continua [71].

\subsubsection{Eringen's model}

Explicitly non-local Eringen's theory holds the primal fields $\left(u_{x}, u_{y}\right)$ and kinematic relations of classical elasticity:

$$
\varepsilon_{i j}^{E}=\frac{1}{2}\left(u_{i, j}+u_{j, i}\right)
$$

For the continuum to be in balance, interactions between material points, that are characterized through traction forces $\left(t_{i}^{E}\right)$, based on Cauchy's theorem, are described in terms of symmetric stress tensor, $\left(\sigma_{i j}^{E}\right)$, and unit normal vector $\left(n_{j}\right)$ :

$$
t_{i}^{E}=\sigma_{i j}^{E} n_{j}
$$

Similar considerations which provided Eq. (3), lead to, in the absence of body forces and non-local residuals,

$$
\sigma_{i j, j}^{E}=0 \text {. }
$$

For a linear elastic and isotropic solid in the domain $\Omega$, the convolution type constitutive relation of integral form of two-phase local/nonlocal Eringen's model is written as $[72,73]$ :

$$
\begin{aligned}
& \sigma_{i j}^{E}=\xi\left(\lambda \varepsilon_{k k}^{E} \delta_{i j}+2 G \varepsilon_{i j}^{E}\right) \\
& +(1-\xi) \int_{\Omega} \tau(r, \kappa)\left(\lambda \varepsilon_{k k}^{E}(\overline{\mathbf{x}}) \delta_{i j}+2 G \varepsilon_{i j}^{E}(\overline{\mathbf{x}})\right) d \Omega(\overline{\mathbf{x}})
\end{aligned}
$$

Here $\xi \in[0,1]$ is the fraction coefficient which controls the weight of local and non-local parts in the constitutive equation. $\tau(r, \kappa)$ denotes the kernel function which accounts for the long-range effects between source point $\mathbf{x}$ and neighbouring points $\overline{\mathbf{x}}$. Kernel function can take many different forms from bell-shaped to conical-shaped as long as the necessity requirements defined by [74] are fulfilled. Considering its advantages in terms of calculation and implementation, a bi-exponential type kernel function is adopted throughout the study:

$$
\tau(r, \kappa)=\frac{e^{-\frac{r}{\kappa}}}{2 \pi^{d-1} \kappa^{d}}
$$

where $d$ is the dimension of the space in which the body of interest is defined, $r$ refers to distance between the two interacting points, and $\kappa$ denotes the nonlocal parameter conveying information about material's internal structure. $r$ generally refers to Euclidean distance; however, in the lack of material continuity, it may also be regarded as the shortest distance between the points inside the body. An alternative strategy to calculate this distance is provided in the subsequent sections of the manuscript.

Eq. (15) may be written in matrix form;

$$
\boldsymbol{\sigma}^{E}=\xi \mathbf{D}^{E} \varepsilon^{E}+(1-\xi) \int_{A} \frac{e^{-\frac{r}{\kappa}}}{2 \pi \kappa^{2}} \mathbf{D}^{E} \varepsilon^{E}(\overline{\mathbf{x}}) d A(\overline{\mathbf{x}})
$$

with following positions, for plane-strain assumption,

$$
\mathbf{D}_{\varepsilon}^{E}=\left[\begin{array}{ccc}
\lambda+2 G & \lambda & 0 \\
\lambda & \lambda+2 G & 0 \\
0 & 0 & G
\end{array}\right]
$$




$$
\boldsymbol{\sigma}^{E}=\left\{\begin{array}{lll}
\sigma_{11}^{E} & \sigma_{22}^{E} & \sigma_{12}^{E}
\end{array}\right\}^{T}, \varepsilon^{E}=\left\{\begin{array}{lll}
\varepsilon_{11}^{E} & \varepsilon_{22}^{E} & 2 \varepsilon_{12}^{E}
\end{array}\right\}^{T}
$$

Cauchy continua is recovered for $\xi=1$ or very small values of $\kappa / L \approx 0$ where $L$ refers to the characteristic length of the structure at macro scale.

\section{Incorporation of geodetical path.}

The use of geodetical path concept was introduced by [66] as a refinement to the Eringen's non-local model. The motivation was to incorporate the deteriorating effect of cracks, holes, incisions and re-antrent boundaries on longrange interactions.

According to [66], the assumption on "non-locality effects propagate in all directions via straight lines (i.e. Euclidean path)" fails in the presence of geometric anisotropy (lack of material continuity). This inadequacy was handled by admitting the use of geodetical path concept. Correspondingly, the distance measure $r$ appeared in kernel function is proposed to be defined as the length of the shortest interior path not intersecting the boundary surfaces of the body. Hence, $r$, equals to Euclidean distance if and only if the straight line joining corresponding pair $((\mathbf{x}, \overline{\mathbf{x}}) \in V)$ does not transit any boundary surface $\partial V$; $r(\mathbf{x}, \overline{\mathbf{x}})=|\mathbf{x}-\overline{\mathbf{x}}|$, while $r(\mathbf{x}, \overline{\mathbf{x}}) \geq|\mathbf{x}-\overline{\mathbf{x}}|$ holds for any nonconvex domain.

In a recent paper [43], a similar approach is followed for a problem involving central crack. In that study, distorting effect of the crack on diffusion process is simply accounted by intercepting the long-range interactions traversing it. However, for more complex structures with multiple holes and curved outer boundaries, a robust algorithm that automatically establishes the geodetic path between any point pair is required [75].

Considering the herein presented example with an elliptic hole of different aspect ratios, which can be considered as a generalisation of the problem treated in an authors' previous article [56], the formulation of Eringen's model is customised to include the geodetical distance into the kernel function. Accordingly, for any two points in a domain that the diffusion process cannot follow a straight line, geodetical path will be calculated by following the steps described below.

Let us assume points $M$ and $N$, with coordinates $\mathbf{x}_{M}=$ $\left\{x_{M}, y_{M}\right\}$ and $\mathbf{x}_{N}=\left\{x_{N}, y_{N}\right\},\left(\mathbf{x}_{M}, \mathbf{x}_{N}\right) \in V$, as the corresponding pair between which the geodetical path is to be calculated, while elliptic hole is centred at the origin with a minor radius $b$ and major radius $a$ (see Fig. 1).

1. After determining whether the Euclidean path traverses the boundary surface of the elliptic hole as in Fig. 1, the algorithm detects tangents to the ellipse from points $M$ and $N$ by exploiting the equivalency between slope of the tangent and derivative of the elliptic curve at corresponding tangent point:

$$
\frac{y-y_{M(N)}}{x-x_{M(N)}}=-\frac{a^{2} x}{b^{2} y}
$$

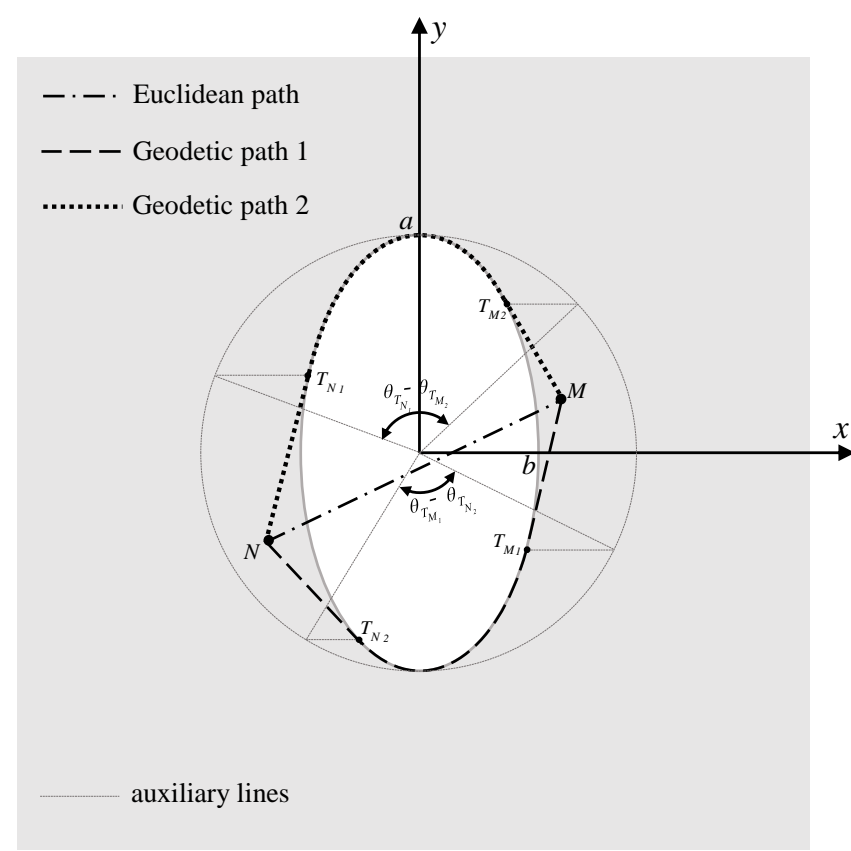

Figure 1: Illustration of geodetic and Euclidean paths for points $M$ and $N$.

Pairs of $x$ and $y$ that satisfy Eq. (20) and the following equation of the ellipse:

$$
\frac{x^{2}}{b^{2}}+\frac{y^{2}}{a^{2}}=1
$$

indicate each tangent points: $\left(\mathbf{x}_{T_{M_{1}}}, \mathbf{x}_{T_{M_{2}}}\right) \in \partial V$ and $\left(\mathbf{x}_{T_{N_{1}}}, \mathbf{x}_{T_{N_{2}}}\right) \in \partial V$.

2. In this step, circumferential length, $s$, between tangent points of opposite pairs is calculated, exploiting the parametric representation of the ellipse:

$$
x=b \cos \theta, y=a \sin \theta
$$

with $\theta$ refers to the eccentric angle at the centre measured from the $x$ axis counter-clockwise. To this end, first, eccentric angles of corresponding points are obtained by substituting their rectangular coordinates into $\theta=\arctan (b y / a x)$, then, the arc length in-between is measured using following equation:

$$
s=\int_{\theta_{1}}^{\theta_{2}} d s=\int_{\theta_{1}}^{\theta_{2}} \underbrace{\left(b^{2} \sin ^{2} \theta+a^{2} \cos ^{2} \theta\right)}_{f(\theta)} d \theta \quad \text { for } \quad \theta_{1}<\theta_{2}
$$

which results in two separate outcomes: $s_{1}$ and $s_{2}$ for each case.

3. In the last step, the geodetical distance to be used in calculations is determined by comparing the total length of both paths: $r=\operatorname{Min}\left(r_{1}, r_{2}\right)$. Here each option includes one arc length and two tangents. For instance; considering the illustration in Fig. 1, geode- 
tical paths are obtained as following:

$$
\begin{aligned}
r_{1(2)} & =\left|\mathbf{x}_{M(N)}-\mathbf{x}_{T_{M_{2}}\left(T_{N_{2}}\right)}\right|+\int_{\theta_{T_{M_{2}}\left(T_{N_{2}}\right)}}^{\theta_{T_{N_{1}}\left(T_{M_{1}}\right)}} f(\theta) d \theta \\
& +\left|\mathbf{x}_{N\left(T_{M_{1}}\right)}-\mathbf{x}_{T_{N_{1}}(M)}\right|
\end{aligned}
$$

With minor adjustments, the presented algorithm and related formulas can be used for any regularly shaped domain including an oval void.

\subsection{Finite element formulations}

In the present study, the FE formulation is derived using the principle of minimum total potential $\Pi$ :

$$
\frac{\partial \Pi[\mathbf{d}]}{\partial \mathbf{d}_{i}}=0, i=1,2, \ldots, N_{\text {total }}
$$

where $\mathbf{d}_{i}$ and $\mathbf{d}$, respectively, denote the nodal displacement vector of $i$ th element and whole model, consisting of $N_{\text {total }}$ elements. Weak non-local character of micropolar (Cosserat) model allows the total potential to be represented as follows

$$
\Pi^{M}[\mathbf{d}]=\sum_{i=1}^{N_{\text {total }}} \Pi_{i}^{M}=\sum_{i=1}^{N_{\text {total }}} \Pi_{i}^{M}\left[\mathbf{d}_{i}^{M}\right]
$$

which, along with Eq. (25), leads to,

$$
\frac{\partial \Pi_{i}^{M}}{\partial \mathbf{d}_{i}^{M}}=0, \quad i=1,2, \ldots N_{\text {total }}
$$

Such a simplified form is not possible for Eringen's nonlocal model due to its strong non-local character accounting for long-range interactions;

$$
\begin{aligned}
\Pi^{E}[\mathbf{d}] & =\sum_{i=1}^{N_{\text {total }}} \Pi_{i}^{E}=\sum_{i=1}^{N_{\text {total }}} \Pi_{i}^{E}\left[\mathbf{d}^{E}\right] \\
& =\sum_{i=1}^{N_{\text {total }}} \Pi_{i}^{E}\left[\mathbf{d}_{1}^{E}, \mathbf{d}_{2}^{E}, \ldots, \mathbf{d}_{N_{\text {total }}}^{E}\right]
\end{aligned}
$$

Correspondingly, for any element number $i$, all the derivatives in Eq. (25) are recovered.

$$
\frac{\partial \Pi_{i}^{E}}{\partial \mathbf{d}_{1}^{E}}=\ldots=\frac{\partial \Pi_{i}^{E}}{\partial \mathbf{d}_{N_{\text {total }}}^{E}}=0, i=1,2, \ldots, N_{\text {total }}
$$

For a domain discretised with four-noded linear quadrilateral elements, Jacobian and inverse Jacobian matrices of each element must be calculated beforehand, for a proper transformation between natural $(\zeta, \eta)$ and physical $(x, y)$ coordinates:

$$
\mathbf{J}_{i}=\left[\begin{array}{cc}
\frac{\partial \sum_{j=1}^{4} N^{j}(\zeta, \eta) x^{j}}{\partial \zeta} & \frac{\partial \sum_{j=1}^{4} N^{j}(\zeta, \eta) y^{j}}{\partial \zeta} \\
\frac{\partial \sum_{j=1}^{4} N^{j}(\zeta, \eta) x^{j}}{\partial \eta} & \frac{\partial \sum_{j=1}^{4} N^{j}(\zeta, \eta) y^{j}}{\partial \eta}
\end{array}\right]_{i}
$$

where $x^{j}$ and $y^{j}$ refer to $x$ and $y$ coordinates of $j$ th node of $i$ th element, while $N^{j}$ denotes the corresponding interpolation function:

$$
\begin{aligned}
& N^{1}=\frac{(1-\zeta)(1-\eta)}{4}, N^{2}=\frac{(1+\zeta)(1-\eta)}{4} \\
& N^{3}=\frac{(1+\zeta)(1+\eta)}{4}, N^{4}=\frac{(1-\zeta)(1-\eta)}{4}
\end{aligned}
$$

\subsubsection{Micropolar (Cosserat) model}

In accordance with the theory, each node possesses three DOFs; in-plane displacements and out-of-plane microrotation, which leads to following nodal unknown vector for an element $e$ :

$$
\begin{aligned}
\mathbf{d}_{e \varepsilon}^{M} & =\left\{\begin{array}{lllll}
\tilde{u}_{x}^{1} & \tilde{u}_{y}^{1} & \ldots & \tilde{u}_{x}^{4} & \tilde{u}_{y}^{4}
\end{array}\right\}_{e}^{T} \\
\mathbf{d}_{e \phi}^{M} & =\left\{\begin{array}{lll}
\tilde{\phi}_{z}^{1} & \ldots & \tilde{\phi}_{z}^{4}
\end{array}\right\}_{e}^{T} \\
\mathbf{d}_{e}^{M} & =\left\{\begin{array}{lll}
\mathbf{d}_{e \varepsilon}^{M} & \mathbf{d}_{e \phi}^{M}
\end{array}\right\}^{T}
\end{aligned}
$$

Here over tilde symbol is used to indicate the nodal values and superscripts refer to the node number. The field variables within the elements are approximated by interpolating the nodal values of corresponding element via linear shape functions,

$$
\begin{gathered}
\mathbf{u}_{e}^{M}(\zeta, \eta)=\mathbf{N}_{u} \mathbf{d}_{e \varepsilon}^{M}, \quad \boldsymbol{\varphi}_{e}(\zeta, \eta)=\mathbf{N}_{\phi} \mathbf{d}_{e \phi}^{M} \\
\mathbf{N}_{u}=\left[\begin{array}{ccccc}
N^{1} & 0 & \ldots & N^{4} & 0 \\
0 & N^{1} & \ldots & 0 & N^{4}
\end{array}\right], \\
\mathbf{N}_{\phi}=\left[\begin{array}{lll}
N^{1} & \ldots & N^{4}
\end{array}\right]
\end{gathered}
$$

The usual procedures of FEM is followed to obtain the formulation. Strain, and curvature fields of the element are expressed in the form:

$$
\begin{aligned}
& \varepsilon_{e}^{M}(\zeta, \eta)=\left[\begin{array}{ll}
\mathbf{L}_{e}^{M} \mathbf{N}_{u} & \mathbf{M} \mathbf{N}_{\phi}
\end{array}\right] \mathbf{d}_{e}^{M}=\mathbf{B}_{e \varepsilon}^{M} \mathbf{d}_{e}^{M} \\
& \chi_{e}(\zeta, \eta)=\left[\begin{array}{ll}
\mathbf{0}_{2 \times 8} & \nabla_{e} \mathbf{N}_{\phi}
\end{array}\right] \mathbf{d}_{e}^{M}=\mathbf{B}_{e \chi}^{M} \mathbf{d}_{e}^{M},
\end{aligned}
$$

where $\mathbf{L}_{e}^{M}, \mathbf{M}$, and $\nabla_{e}$ respectively refer to the differential matrix operator, permutation vector and gradient operator which are, in natural coordinate system,

$$
\begin{gathered}
\mathbf{L}_{e}^{M}=\left[\begin{array}{cc}
\frac{\partial}{\partial \zeta} J_{11}^{-1}+\frac{\partial}{\partial \eta} J_{12}^{-1} & 0 \\
0 & \frac{\partial}{\partial \zeta} J_{21}^{-1}+\frac{\partial}{\partial \eta} J_{22}^{-1} \\
\frac{\partial}{\partial \zeta} J_{21}^{-1}+\frac{\partial}{\partial \eta} J_{22}^{-1} & 0 \\
0 & \frac{\partial}{\partial \zeta} J_{11}^{-1}+\frac{\partial}{\partial \eta} J_{12}^{-1}
\end{array}\right]_{e} \\
\mathbf{M}=\left[\begin{array}{c}
0 \\
0 \\
+1 \\
-1
\end{array}\right], \nabla_{e}=\left[\begin{array}{c}
\frac{\partial}{\partial \zeta} J_{11}^{-1}+\frac{\partial}{\partial \eta} J_{12}^{-1} \\
\frac{\partial}{\partial \zeta} J_{21}^{-1}+\frac{\partial}{\partial \eta} J_{22}^{-1}
\end{array}\right]_{e}
\end{gathered}
$$

Inserting Eqs. (35) into (8), with positions in Eq. (10), the work-conjugated stress and couple-stress become as follows,

$$
\begin{aligned}
& \boldsymbol{\sigma}_{e}^{M}(\zeta, \eta)=\mathbf{D}_{e \varepsilon}^{M} \mathbf{B}_{e \varepsilon}^{M}(\zeta, \eta) \mathbf{d}_{e}^{M} \\
& \boldsymbol{\mu}_{e}^{M}(\zeta, \eta)=\mathbf{D}_{e \chi}^{M} \mathbf{B}_{e \chi}^{M}(\zeta, \eta) \mathbf{d}_{e}^{M}
\end{aligned}
$$


Lastly, with substituting the elastic strain energy

$$
U_{m}^{M}=\frac{h}{2} \int_{-1}^{1} \int_{-1}^{1}\left(\left(\varepsilon_{m}^{M}\right)^{T} \boldsymbol{\sigma}_{m}^{M}+\left(\boldsymbol{\chi}_{m}\right)^{T} \boldsymbol{\mu}_{m}\right) \operatorname{det}\left|\mathbf{J}_{m}\right| d \zeta d \eta
$$

and work potential $\left(W_{m}^{M}\right)$ of an element $\left(\Pi_{m}^{M}=U_{m}^{M}+\right.$ $\left.W_{m}^{M}\right)$ into the Eq. (27), the element formulation is derived:

$$
\begin{aligned}
\mathbf{f}_{m}^{M} & =\left(\mathbf{k}_{m \varepsilon}^{M}+\mathbf{k}_{m \chi}^{M}\right) \mathbf{d}_{m}^{M} \\
\mathbf{k}_{m \varepsilon}^{M} & =h \int_{-1}^{1} \int_{-1}^{1}\left(\mathbf{B}_{m \varepsilon}^{M}\right)^{T} \mathbf{D}_{m \varepsilon}^{M} \mathbf{B}_{m \varepsilon}^{M} \operatorname{det}\left|\mathbf{J}_{m}\right| d \zeta d \eta \\
\mathbf{k}_{m \chi}^{M} & =h \int_{-1}^{1} \int_{-1}^{1}\left(\mathbf{B}_{m \chi}^{M}\right)^{T} \mathbf{D}_{m \chi}^{M} \mathbf{B}_{m \chi}^{M} \operatorname{det}\left|\mathbf{J}_{m}\right| d \zeta d \eta
\end{aligned}
$$

The above-given integrations require a numerical integration scheme, such as Gauss Quadrature Method. As per usual practice in FEM, $2 \times 2$ Gauss points provide sufficient accuracy for the linear element used herein, and resulting in following formulation:

$\mathbf{k}_{m}^{M}=h \sum_{p=1}^{2} \sum_{r=1}^{2} w_{p} w_{r} \mathbf{B}_{m}^{M}\left(\zeta_{p}, \eta_{r}\right)^{T} \mathbf{D}_{m}^{M} \mathbf{B}_{m}^{M}\left(\zeta_{p}, \eta_{r}\right) \operatorname{det}\left|\mathbf{J}_{m}\right|$

where subscripts $p$ and $r$ refer to components of either weights $(w) \quad\{1.0,1.0\}$ or coordinates $(\zeta, \eta)$ $\{-1 / \sqrt{3},+1 / \sqrt{3}\}$.

\subsubsection{Eringen's model}

FE formulation of a solid obeying Eringen's constitutive equations admits identical degrees of freedom, and strain interpolation over each element to those of classical theory.

$$
\begin{aligned}
& \mathbf{d}_{e}^{E}=\left\{\begin{array}{lllll}
\tilde{u}_{x}^{1} & \tilde{u}_{y}^{1} & \ldots & \tilde{u}_{x}^{4} & \tilde{u}_{y}^{4}
\end{array}\right\}_{e}^{T} \\
& \mathbf{u}_{e}^{E}(\zeta, \eta)=\mathbf{N}_{u} \mathbf{d}_{e}^{E} \\
& \boldsymbol{\varepsilon}_{e}^{E}(\zeta, \eta)=\mathbf{L}_{e}^{E} \mathbf{N}_{u} \mathbf{d}_{e}^{E}=\mathbf{B}_{e}^{E} \mathbf{d}_{e}^{E}
\end{aligned}
$$

where differential matrix operator for an element $e$ takes the following form in natural coordinate system:

$$
\mathbf{L}_{e}^{E}=\left[\begin{array}{cc}
\frac{\partial}{\partial \zeta} J_{11}^{-1}+\frac{\partial}{\partial \eta} J_{12}^{-1} & 0 \\
0 & \frac{\partial}{\partial \zeta} J_{21}^{-1}+\frac{\partial}{\partial \eta} J_{22}^{-1} \\
\frac{\partial}{\partial \zeta} J_{21}^{-1}+\frac{\partial}{\partial \eta} J_{22}^{-1} & \frac{\partial}{\partial \zeta} J_{11}^{-1}+\frac{\partial}{\partial \eta} J_{12}^{-1}
\end{array}\right]_{e}
$$

On the other hand, due to convolution type constitutive equation of Eringen's theory (Eq. (17)), the relation between elemental stress and strain requires more attention. Indeed, a close look to kernel function reveals that the long-range interactions practically vanish beyond a certain limit. This limit is called as influence zone, and eliminates the necessity to consider the interaction of all elements
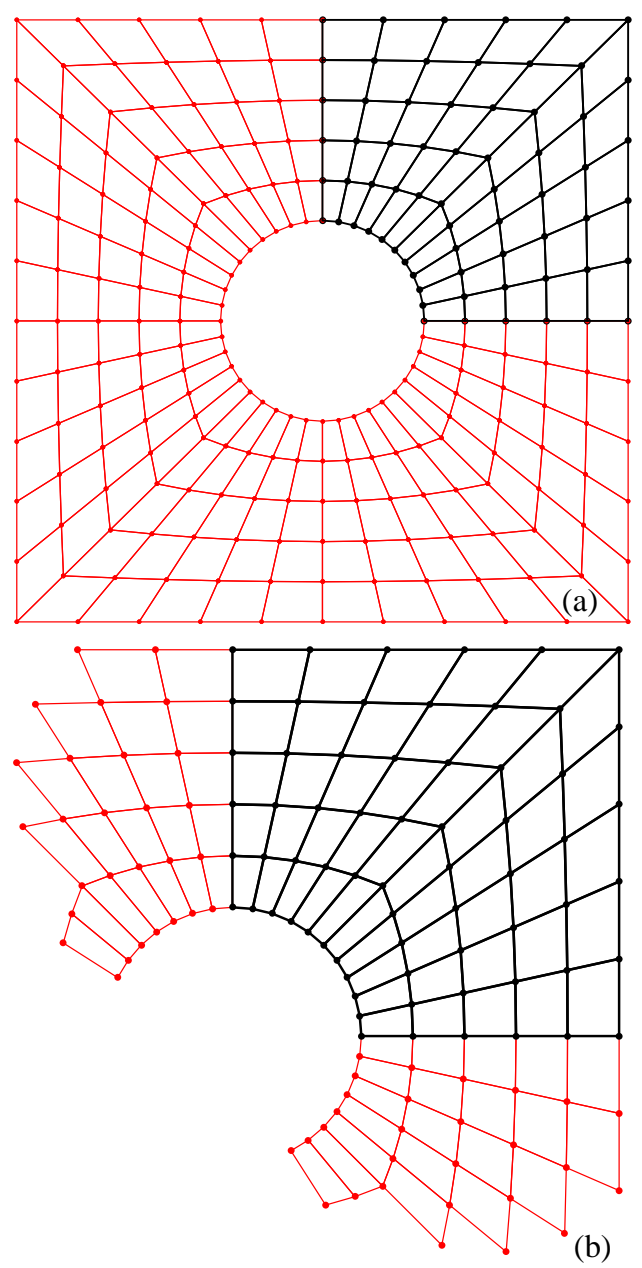

Figure 2: (a) First and (b) final steps of formation of nonconventional symmetric FE model for a sample problem with $L / a=3.0$ and $b / a=1.0$ (red: padding region, black: conventional quarter symmetric model).

with each other. This situation provides a great advantage in computational terms, limiting the number of elements which contributes to the stress of a generic $e^{\text {th }}$ element. Therefore, the constitutive relation becomes,

$$
\begin{aligned}
& \boldsymbol{\sigma}_{e}^{E}(\zeta, \eta)=\xi_{e} \mathbf{D}_{e}^{E} \mathbf{B}_{e}^{E}(\zeta, \eta) \mathbf{d}_{e}^{E}+ \\
& \left(1-\xi_{e}\right) \sum_{i \in R_{I_{e}}} \int_{-1}^{1} \int_{-1}^{1} \frac{e^{-\frac{r}{\kappa}}}{2 \pi \kappa^{2}} \mathbf{D}_{i}^{E} \overline{\mathbf{B}}_{i}^{E}(\bar{\zeta}, \bar{\eta}) \mathbf{d}_{i}^{E} \operatorname{det}\left|\overline{\mathbf{J}}_{i}\right| d \bar{\zeta} d \bar{\eta}
\end{aligned}
$$

where the over bar indicates that the corresponding matrix is written in terms of $\bar{\zeta}, \bar{\eta}$, while $R_{I_{e}}$ refers to the list of elements that fall into the influence zone of $e^{\text {th }}$ element (Please see Fig. 2 in [55]). Inserting the constitutive relation in (43) into the elastic strain energy of an element, which is,

$$
U_{m}^{E}=\frac{h}{2} \int_{-1}^{1} \int_{-1}^{1}\left(\varepsilon_{m}^{E}\right)^{T} \boldsymbol{\sigma}_{m}^{E} \operatorname{det}\left|\mathbf{J}_{m}\right| d \zeta d \eta
$$


and performing derivations in Eq. (29), following element formulation is obtained.

$$
\begin{aligned}
& \mathbf{f}_{m}^{E}=\xi_{m} \mathbf{k}_{m}^{E} \mathbf{d}_{m}^{E}+2\left(1-\xi_{m}\right) \mathbf{k}_{m m}^{E} \mathbf{d}_{m}^{E} \\
& +\left(1-\xi_{m}\right) \sum_{n \in R_{I_{m}}} \mathbf{k}_{m n}^{E} \mathbf{d}_{n}^{E}+\left(1-\xi_{n}\right) \sum_{n \in R_{I_{m}}}\left(\mathbf{k}_{n m}^{E}\right)^{T} \mathbf{d}_{n}^{E}
\end{aligned}
$$

Here third and fourth terms on the right side represent the long-range effects such that third term emerges due to contribution of other elements to $m$ th element, while fourth term appears by virtue of $m$ th element's contribution to others.

$$
\begin{aligned}
& \mathbf{k}_{m}^{E}=h \int_{-1}^{1} \int_{-1}^{1}\left(\mathbf{B}_{m}^{E}\right)^{T} \mathbf{D}_{m}^{E} \mathbf{B}_{m}^{E} \operatorname{det}\left|\mathbf{J}_{m}\right| d \zeta d \eta, \\
& \mathbf{k}_{m n}^{E}=\frac{h}{2} \int_{-1}^{1} \int_{-1}^{1} \int_{-1}^{1} \int_{-1}^{1} \mathbf{K}_{m n} \operatorname{det}\left|\overline{\mathbf{J}}_{n}\right| \operatorname{det}\left|\mathbf{J}_{m}\right| d \bar{\zeta} d \bar{\eta} d \zeta d \eta, \\
& \mathbf{K}_{m n}=\frac{e^{-\frac{r}{\kappa}}}{2 \pi \kappa^{2}}\left(\mathbf{B}_{m}^{E}\right)^{T} \mathbf{D}_{n}^{E} \overline{\mathbf{B}}_{n}^{E}
\end{aligned}
$$

It is clear from expression in Eq. $(46)_{2}$ that, for a homogeneous solid, as in this study, following relations hold:

$$
\xi_{m}=\xi_{n}, \quad\left(\mathbf{k}_{n m}^{E}\right)^{T}=\mathbf{k}_{m n}^{E}
$$

The integrations are obtained using Gauss Quadrature Method. This time, the numbers of Gauss sampling points should be decided upon $\kappa / l_{e}$ on which the functions to be integrated highly depend. As this ratio decreases, the number of Gauss points should be increased due to increasing gradient of the integrand, to reduce numerical errors. Corresponding integration procedure for $\mathbf{k}_{m n}^{E}$ is provided below.

$$
\begin{aligned}
\mathbf{k}_{m n}^{E}= & \frac{h}{2} \sum_{p=1}^{N Q_{m}} \sum_{r=1}^{N Q_{m}} \sum_{s=1}^{N Q_{n}} \sum_{q=1}^{N Q_{n}} w_{p} w_{r} w_{s} w_{q} \frac{e^{-\frac{r}{\kappa}}}{2 \pi \kappa^{2}} \\
& \mathbf{B}_{m}^{E}\left(\zeta_{p}, \eta_{r}\right)^{T} \mathbf{D}_{n}^{E} \overline{\mathbf{B}}_{n}^{E}\left(\bar{\zeta}_{s}, \bar{\eta}_{q}\right) \operatorname{det}\left|\overline{\mathbf{J}}_{n}\right| \operatorname{det}\left|\mathbf{J}_{m}\right|
\end{aligned}
$$

where $N Q_{m}$ and $N Q_{n}$ indicate the number of Gauss points in $m$ th and $n$th elements, respectively, while $r$ equals to either Euclidean or geodetical distance between corresponding Gauss sampling points.

\subsubsection{Formation of symmetric FE models}

Exploiting the symmetry in finite element analysis is favoured due to computational concerns. With a convenient symmetry model, the total number of degree of freedoms is drastically reduced, yet the identical results with full model would be obtained. Considering our example problem, quarter symmetric FE models of Cauchy and micropolar (Cosserat) plates can simply be achieved by submitting the appropriate boundary conditions. Although Cauchy plate does not require any further constraint (other than those already mentioned), the micro

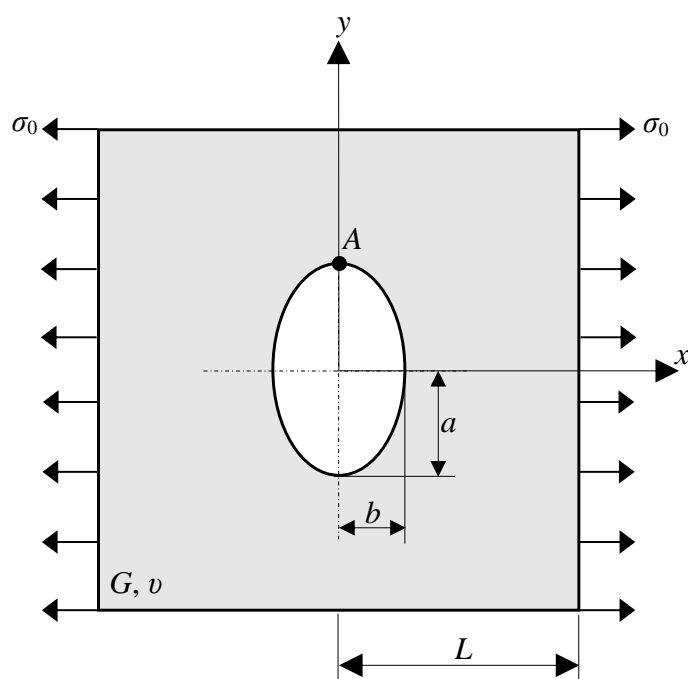

Figure 3: Illustration of example problem.

rotations of nodes located at $x=0$ and $y=0$ should be restricted for micropolar plate. However, for plate models conducting Eringen's theory, the existence of long-range effects leads to a non-conventional type symmetry model. As the shortcoming of conventional symmetric model is the absence of neighbour elements that should have interact with the elements in quarter portion due to non-local effects, the problem can be tackled by adapting the concept of padding region to the FE model. Padding region is originally introduced for concurrent partitioned-domain multiscale models to provide a full neighbour environment to the sub-atomic domain [76]. Herein we consider it as a continuum region with imaginary padding elements, whose energy is not explicitly included in the energy functional, but deformation is traced to properly build the cross stiffness matrices $\mathbf{k}_{m n}$ of real elements in quarter model. The dimension of padding region is directly related to nonlocality of model, and can be easily determined by following a two-step algorithm:

1. First, all elements in the remaining three quarter of the full model are assumed as possible candidates for padding region (Fig. 2(a)).

2. Then, padding elements that do not interact with any of the real elements are removed from the model (Fig. $2(\mathrm{~b}))$.

Although the presence of padding elements may seem to increase the total DOFs, in fact, the global equation system submitted to the solver has the same dimensions with conventional quarter symmetry model because of the existence of multi freedom constraints (MFCs). In general, depending on the level of complexity of the constraints, different techniques are available to treat them [77-79], while for homogeneous and linear constraints, the master-slave approach [80] can be conducted. In master-slave approach, an auxiliary equation system is generated to represent the kinematic relation between master and slave nodes. This 

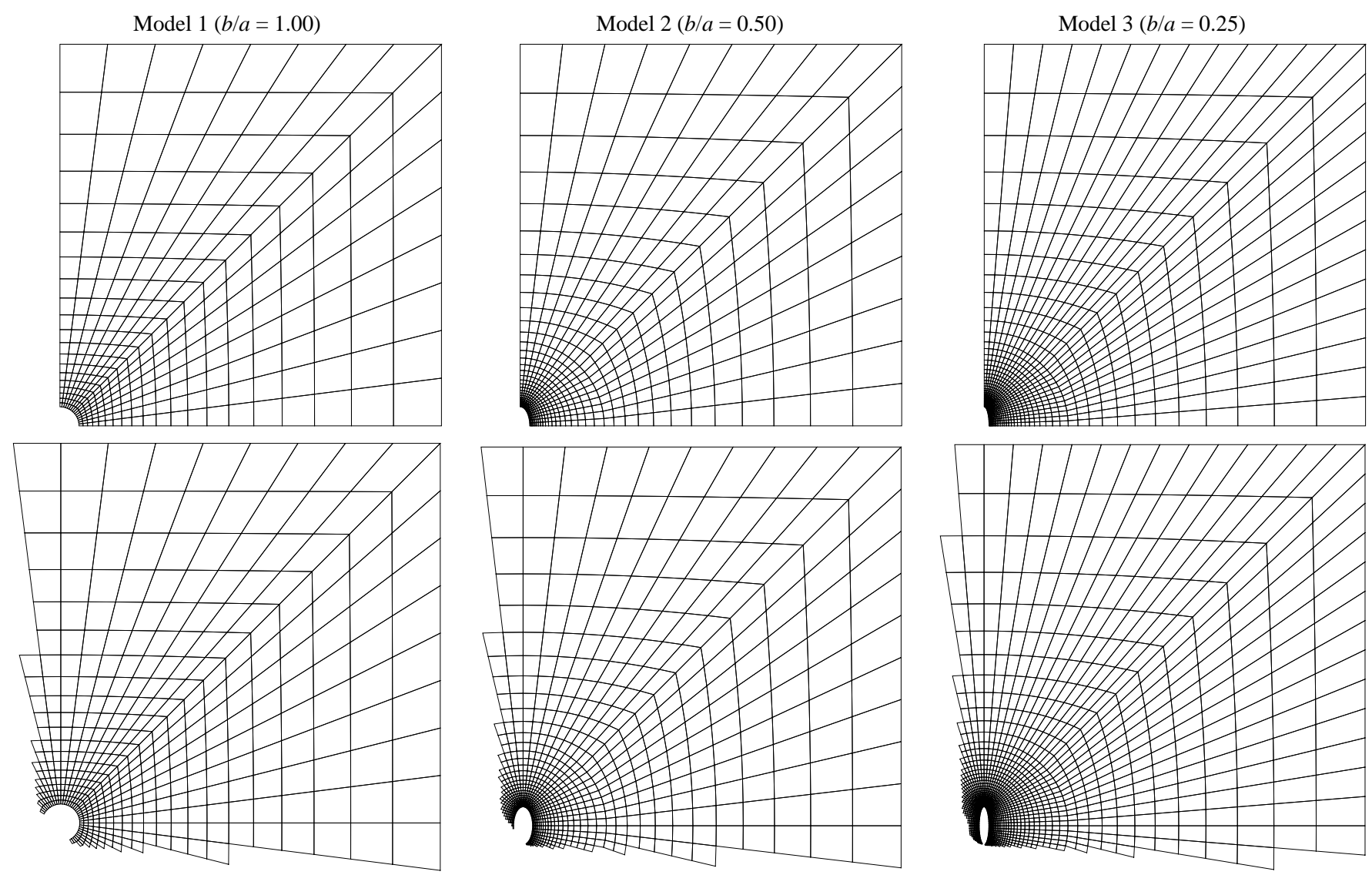

Figure 4: Mesh configuration of quarter symmetry FE models for Cauchy, micropolar (Cosserat) (top) and Eringen's (bottom) plates.

linear system ensures that any node at padding region displaces in accordance with the corresponding node in quarter model.

$$
\mathbf{d}=\mathbf{T} \hat{\mathbf{d}}
$$

Here $\hat{\mathbf{d}}$ and $\mathbf{d}$ respectively denote the nodal displacements of quarter (conventional) and enlarged (nonconventional) symmetric models. Due to simplicity of the symmetry conditions, the matrix $\mathrm{T}$ consists of 0 and \pm 1 . This matrix is then used for congruential transformation of the model to produce the modified system of equations:

$$
\begin{aligned}
& \hat{\mathbf{K}}=\mathbf{T}^{T} \mathbf{K} \mathbf{T} \\
& \hat{\mathbf{f}}=\mathbf{T}^{T} \mathbf{f} \\
& \hat{\mathbf{d}}=\hat{\mathbf{K}}^{-1} \hat{\mathbf{f}}
\end{aligned}
$$

With this technique, the global stiffness equations are reduced to a form: $\hat{\mathbf{K}} \hat{\mathbf{d}}=\hat{\mathbf{f}}$ having same dimensions with the quarter symmetry model (therefore the solution phase requires quite similar computational effort to that of Cauchy plate). Nevertheless, the main advantage of this technique is the reduced time needed to form the global stiffness matrix, due to decreased number of long-range interactions. For instance, for the model illustrated in Fig. 2(b), the number of cross stiffness matrices, $\mathbf{k}_{m n}$, to be calculated are $69.6 \%$ less than its full model. Resemblances between the proposed nonconventional symmetry model and the one recently presented by [65] are easy to observe. In the work of [65], an iterative approach is proposed to satisfy the constraints between master and slave nodes for a uniformly meshed model.

The nonconventional (enlarged) symmetric model of the present study is validated by comparing displacement/stress fields of the model in Fig. 2(b), with the corresponding full model in [56], and identical results are obtained.

\section{Numerical simulations}

In this section, a comparison between local Cauchy, 'implicitly/weakly' non-local micropolar (Cosserat) and 'explicitly/strongly' non-local Eringen's models is made through an example problem of practical importance: an infinite plate weakened with a central elliptical hole.

As illustrated in Fig. 3, the square shaped, linear, elastic plate has an edge length of $2 L$, while the semi-major and semi-minor axes of the ellipse are denoted as $a$ and $b$, respectively. To be in accordance with authors previous paper; [55], the fixed radius $a$ equals to $0.05 \mathrm{~m}$, while the edge length is assumed sufficiently large (i.e. $L / a=20.0$ ), approximating the infinite plate. The simulations are repeated for three different values of minor radius; $b / a=1.0$ 


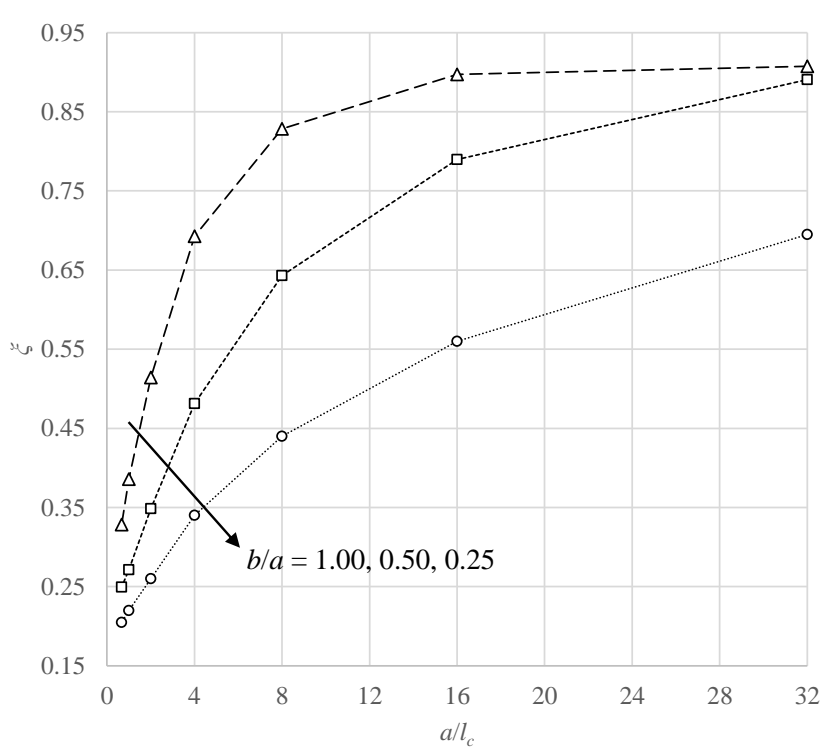

Figure 5: Relation between fraction coefficient, $\xi$, in Eringen's model and non-dimensionalised internal characteristic length, $a / l_{c}$, in micropolar model, yielding identical stress concentration factors for various aspect ratios $(b / a): 1.0$ (triangle), 0.5 (square) and 0.25 (circle).

(Model 1), $b / a=0.5$ (Model 2), $b / a=0.25$ (Model 3) to study the effect of non-locality on sharp edges. The uniform tensile stress subjected parallel to the minor axis of the ellipse has a magnitude of $\sigma_{0}=100 \mathrm{MPa}$, while the vertical displacement of $x$ axis $(v(x, 0)=0)$ and horizontal displacement of $y$ axis $(u(0, y)=0)$ are restricted to impose the essential boundary conditions.

The FE analysis are performed by further submitting the symmetry related boundary conditions introduced in previous section. To capture the increasing curvature of the ellipse, the spatial discretisation at the vicinity of the hole is increased for decreasing ratios of $b / a$, which leads to 320 elements (and 357 nodes), 576 elements (and 627 nodes) and 1040 elements (and 1107 nodes) for Models 1, 2 and 3, respectively (see Fig. 4). For mesh sensitivity analysis the results of Model 1 are compared with [56] considering same material parameters. Although, the number of elements in the present study is increased by $25 \%$ with respect to the ones in [56], the stress concentration factor at point $\mathrm{A}$, (i.e. $\left.\mathrm{SCF}=\left(\sigma_{x x}\right)_{A} / \sigma_{0}\right)$ only changed by $1.4 \%$. Note that, stresses are recovered by $d i$ rect evalution method, that is, calculating the stresses directly at the nodes for each element, and averaging them in nodes shared by multiple elements. For sufficiently dense discretisation, this technique does not bring considerable amount of numerical error than extrapolation method, and it is much easier to employ. Considering Cauchy model, SCF is recovered slightly higher than analytical solution (e.g. the relative difference with exact solution [64] is about $9 \%$ for $b / a=1.0$ ) due to the use of linear elements in a region with high stress gradient. Note that this situation is not the same in non-local models (e.g. the relative

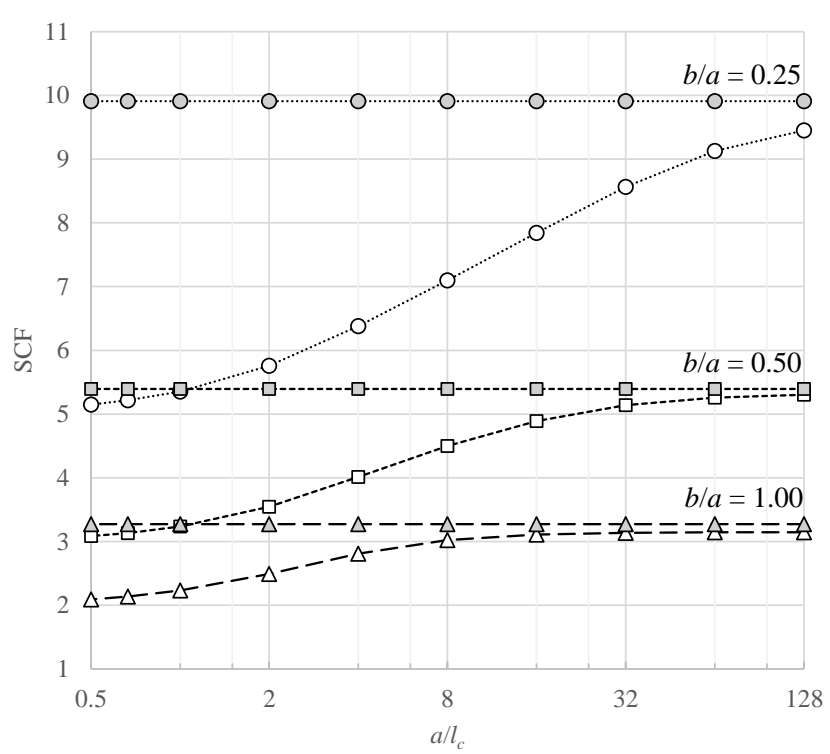

Figure 6: Variation of stress concentration factor of local Cauchy (grey) and non-local micropolar (Cosserat) and Eringen's models (white) considering different internal characteristic lengths (or fraction coefficients) for various aspect ratios $(b / a)$.

difference with exact solution [57] is about $3 \%$ for implicitly non-local plate with $b / a=1.0)$, whether implicit or explicit, since the stress distribution is relatively smooth by virtue of non-locality [55].

For all the calculations, Poisson's ratio and shear modulus are assumed as $\nu=1 / 3$, and $G=1.0 \mathrm{GPa}$. For micropolar plate, the coupling number is $N=0.9$, while different values of internal characteristic length are considered in the interval $l_{c} / a \in[1 / 128,2]$. To have a reasonable comparison between micropolar (Cosserat) and Eringen's non-local models, an equivalency in terms of a characteristic quantity between the two is looked for. Among many alternatives, SCF seems to be a reasonable candidate for this purpose, especially in computational terms [55]. To this end, for each aspect ratio, $(b / a)$, and internal characteristic length of 'implicit' model $\left(a / l_{c}\right)$ considered herein, fraction coefficient $(\xi)$ of 'explicit' model is tailored to have SCFs in accordance with the 'implicit' one, with the following objective function to be minimized either by trial and error or by an optimization technique, such as; Differential Evolution Method (DEM) $[81]^{2}$ :

$$
f(\xi)=\left|\frac{S C F_{E}(\xi)}{S C F_{M}}-1\right|
$$

As clearly seen, the tuning of the non-locality of Eringen's model is performed through the fraction coefficient $\xi \in[0,1]$ for an arbitrarily chosen, yet reasonable nonlocal parameter; $\kappa=0.2 a$ [39]. Although tailoring nonlocal parameter instead of fraction coefficient is an option, it is not favoured due to computational concerns.

\footnotetext{
${ }^{2}$ For the details, the readers are referred to [56] and [82].
} 


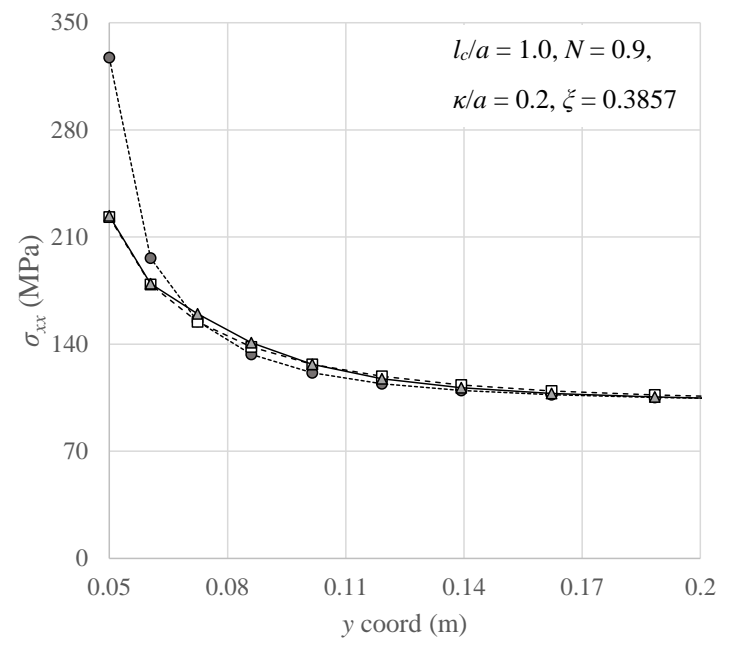

(a)

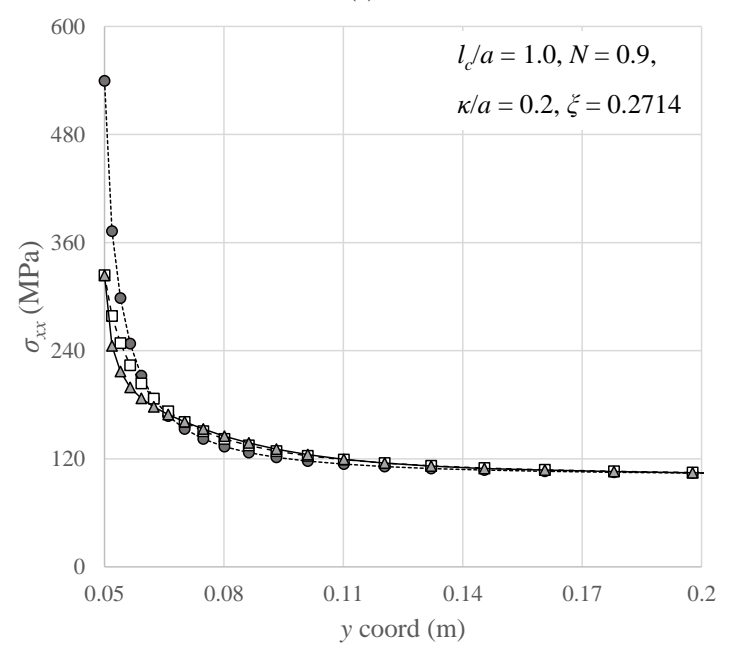

(b)

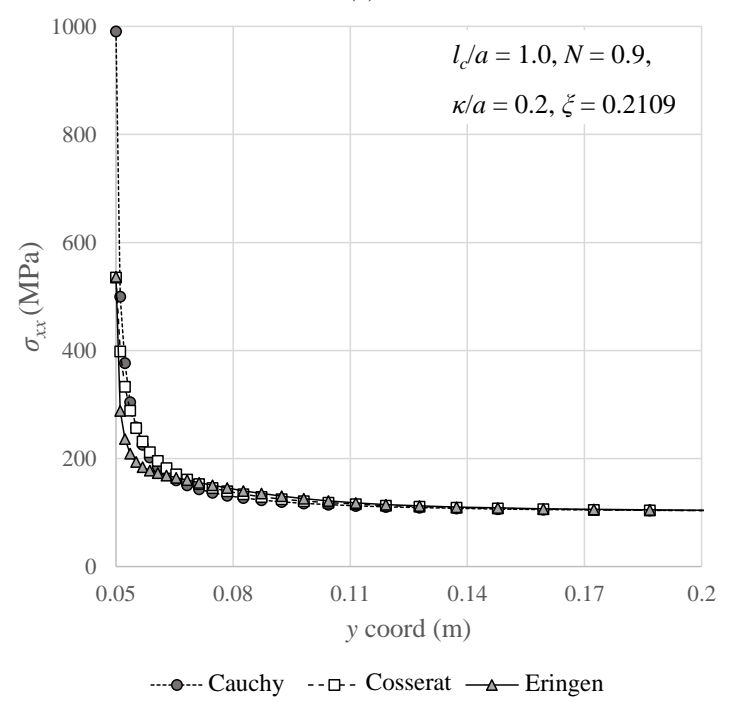

(c)

Figure 7: Stress variation along $y$ axis for (a) $b / a=1.0$, (b) $b / a=$ 0.5, (c) $b / a=0.25$
Fig. 5 presents the relation between internal characteristic length $\left(l_{c}\right)$ in micropolar model and fraction coefficient $(\xi)$ in Eringen's model which leads to equal SCF. A dependence of these iso-SCF curves on aspect ratio is observed, which leads to the conclusion that having a unified value for fraction coefficient, $\xi$, that is acceptable for all aspect ratios could not be obtained, at least for the nonlocal parameter $\kappa=0.2 a$, since the dimensions of the hole strongly affects the stiffness of Eringen's model: for a fixed non-local parameter, $\kappa$, and fraction coefficient, $\xi$, a decrease in aspect ratio corresponds to less missing neighbour relations for the elements located around the vicinity of the hole; hence, a stiffer structure. That is why, the non-locality of Eringen's model has to be tuned by increasing the contribution of non-local part in two-phase constitutive relation to end up with a softer plate that yields identical SCFs with corresponding micropolar one.

Variation of SCF with non-locality for different aspect ratios are illustrated in Fig. 6. Note that, even if the horizontal axis (in log scale) denotes the non-dimensionalised internal characteristic length $a / l_{c}$ in micropolar model, it may be replaced with the equivalent fraction coefficient $\xi$ in Eringen's model, with the aid of Fig. 5. For small values of $a / l_{c}$ (or $\xi$ ), the non-local effects are more pronounced and SCF are much less than those estimated by local elasticity. It should be noted here that even if the resulting SCF are equal in both non-local models, a difference in stress distribution might be expected, and will be demonstrated. For micropolar model, as the ratio $a / l_{c}$ increases, the SCF converges to a value which is close (but not equal since $N=0.9$ ) to those of local elasticity.

In calculation of long-range interactions in Eringen's theory, geodetic distance, which covers $0.81 \%$ (for $b / a=$ 1.0 ), $10.56 \%$ (for $b / a=0.5$ ) and $19.04 \%$ (for $b / a=0.25$ ) of cross stiffness matrices is adopted. However, the numerical experiences of the authors reveal that using geodetic distance does not affect the numerical results considerably for the problem at hand: even for highest non-locality (i.e. $\kappa=0.2 a, \xi=0.205$ ) and least aspect ratio (i.e. $b / a=0.25)$, the difference between the SCFs, obtained using Euclidean and geodetic distance measures, is only $0.18 \%$. This is because the radius of influence zone for considered non-local parameter is comparable with the dimensions of the ellipse. Therefore, the conclusion of using Euclidean and geodetical distance lead to similar numerical results is limited with the problems and numerical parameters considered in this study.

In Fig. 7, alteration of normal stress $\sigma_{x x}$ along $y$ axis is plotted for a fixed internal characteristic length $a / l_{c}=1.0$ (in micropolar theory), and its correspondent fraction coefficients $\xi=0.3857,0.2714,0.2109$ (in Eringen's theory) for various aspect ratios $(b / a=1.0,0.5,0.25)$. Note that, if attaining a unified relation between internal characteristic length $l_{c}$ and fraction coefficient $\xi$ that satisfies all aspect ratios were possible, the curves in Fig. 5 would coincide, and thereby fraction coefficient, $\xi$, would take a single value for each $a / l_{c}$. It is clearly seen from the figure that, 
SCF of both non-local models begin with the same value as they are forced to do so with the aid of Eq. (51), while local case always provides higher maximum stress. However, for $y>0$ a sharper decrease, which becomes more severe for smaller aspect ratios, is observed for Eringen's theory. Such a behaviour is attributed to increased nonlocality of Eringen's model in order to keep its maximum stress value in accordance with the corresponding micropolar plate as suggested by Fig.5. As an inevitable outcome of more pronounced non-local character, the overall Eringen's plate is softened leading to better load distribution capacity. Indeed, for $b / a=0.5,0.25$, Eringen's estimation of stress is less than those of Cauchy and micropolar up to $y \approx 0.065 \mathrm{~m}$, while the results of all theories become very close to each other for $y>0.15 \mathrm{~m}$. This is also evident from distribution of normal stress component $\sigma_{x x}$ that is plotted in Fig. 8 for various aspect ratios. The left half of these figures corresponds to micropolar (Cosserat) solution and the right halves are the estimations of Eringen's model solution, which provides identical SCF to micropolar model. Note that this is a close-up look around the elliptic hole, for which the boundary effects (explained in [55]) in Eringen's model vanishes. As expected by previous discussions, an equivalency at a single point increases the difference of stress distributions on the region, especially for sharper holes.

\section{Final Remarks}

According to the classification of [12], [14], and [15], adopted in [17], non-local theories can be considered as 'implicit/weak' and 'explicit/strong' based on the perception of internal length parameters through which the information of underlying material organization is preserved. In the present paper, a comparison between the response of local (Cauchy) and non-local models, both of 'implicit', micropolar (Cosserat), and 'explicit', Eringen's, type, in the presence of geometric singularities is provided, by means of an example problem of practical importance: an infinite weakened with a central elliptic hole. The solutions have been obtained using finite elements (FE) models, specifically formulated to treat the two non-local cases. In the case of Eringen's model in particular, the procedure is customised by adopting geodetical distance, instead of Euclidean one, in order to account for the distorting effect of the hole on diffusion process. For the sake of computational efficiency, conventional and non-conventional (enlarged) symmetric FE models have been adopted for Cauchy, micropolar and Eringen plates, respectively. The incorporation of geodetical distance alongside with a validated symmetric finite element model allowed the problem to be examined in the framework of Eringen's nonlocal model, for the first time. Different aspect ratios $(b / a)$ for the hole, and parameters $\left(l_{c}, \xi\right)$ both introducing nonlocality to the two models, respectively, have been considered. As a key feature, the criterion to search for an equivalency between the two non-local models is chosen to
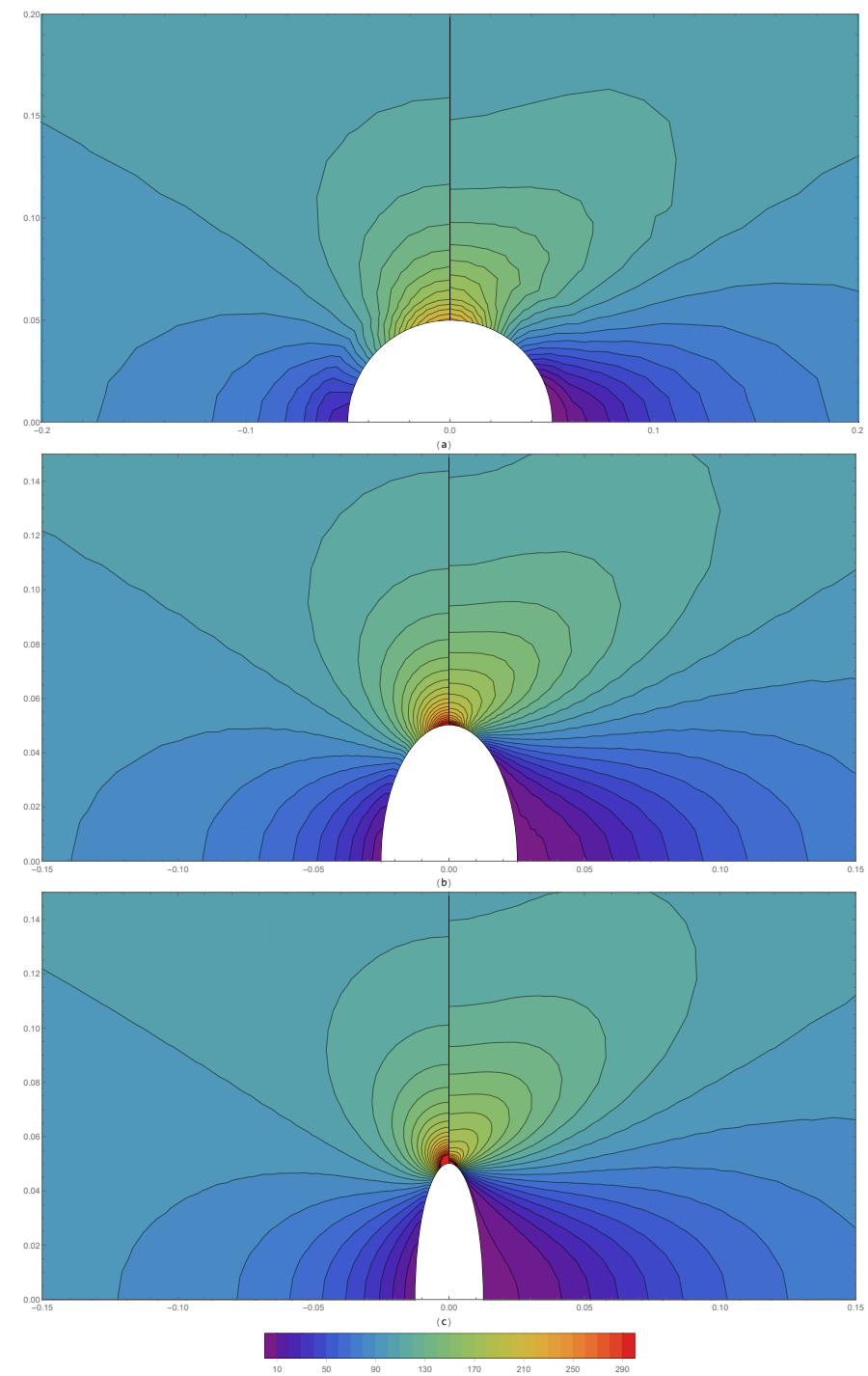

Figure 8: Zoom-in looks of contour plots of normal stress field of micropolar (Cosserat) (left) and Eringen (right) non-local models for (a) $b / a=1.0$, (b) $b / a=0.5$, (c) $b / a=0.25$.

be the stress concentration factor (SCF), the detection of which provides great simplification in computational terms while tuning the fraction coefficient in Eringen's theory. The identification process discloses that the resulting fraction coefficient, $\xi$, for a given internal characteristic length, $l_{c}$, (or visa versa) turns out to be dependent on the aspect ratio of the elliptic hole due to the strong non-local character of Eringen's model, in which case the stiffness of elements around the hole is significantly influenced by missing neighbour relations quantified by the aspect ratio. Correspondingly, increasing non-locality of Eringen's model for sharper holes, in order to keep SCFs identical with its micropolar counterparts leads to softer plates that are able to better distribute the load.

Meanwhile, comparison between local Cauchy and nonlocal micropolar/Eringen's, models emphasizes the importance of adopting non-classical theories for problems with accountable size-effects. Since the classical theory lacks 
the internal length parameters, its prediction of SCF at the tip of the hole is always greater than the one obtained by employing non-classical theories, independent of how they incorporate the size effects. This well-known result of classical theory might be, therefore, misleading for complex microstructured materials and can be corrected by using a non-local model which may either be of implicit or explicit type. However, in computational terms, micropolar model is more effortless compared to Eringen's model, which requires the consideration of long-range interactions for each material point due to its convolution type constitutive equation. This relatively complicated relation between stress and strain measures manifests itself both on integration operations of element stiffness matrices, and formation of the global stiffness matrix.

The present study is expected to offer a different perspective for handling the materials with defects of comparable size of its microstructure by focusing on the advantages of two different non-local approaches although their usual application fields are quite different. The study can be broaden by looking for possible equivalences between non-local models focusing on global response of the structures, or mechanical problems of practical importance for which such equivalences can be sought. Moreover, the calculations can be extended considering other nonlocal theories (e.g. second gradient models), and geometries/materials/problems that indicate a large disparity between models.

\section{Acknowledgements}

Italian Ministry of Education, University and Research PRIN 2017, project 2017HFPKZY (Grant No. B86J16002300001); Sapienza Research Grants "Progetti Medi" 2017 (Grant No. B83C17001440005) and "Progetti Grandi" 2018 (Protocol No. RG1181642E3B3117). This work was done when Meral Tuna was a Visiting Researcher at DISG, Sapienza University of Rome, with financial support of PRIN 2017 (Grant no. B86J16002300001) "Materials with microstructure: multiscale models for the derivation non-local continua and related numerical simulations".

\section{References}

[1] W. Voigt, Theoretische studien über die elasticitätsverhältnisse der kristalle, Abhandlungender Gesellschaft der Wissenschaften zu Göttingen 34 (1887) 1-100.

[2] W. Voigt, Lehrbuch der Kristallphysik, B.G. Teubner, Leipzig, 1910.

[3] H. Poincaré, Leçons sur la Théorie de l'élasticité, Carré, Paris, 1892.

[4] P. Trovalusci, D. Capecchi, G. Ruta, Genesis of the multiscale approach for materials with microstructure, Arch. Appl. Mech. 79 (11) (2008) 981.

[5] D. Capecchi, G. Ruta, P. Trovalusci, From classical to Voigt's molecular models in elasticity, Arch. Hist. Exact Sci. 64 (5) (2010) 525-559.

[6] D. Capecchi, G. Ruta, P. Trovalusci, Voigt and Poincaré's mechanistic-energetic approaches to linear elasticity and suggestions for multiscale modelling, Arch. Appl. Mech. 81 (11) (2011) 1573-1584.
[7] P. Trovalusci, Discrete to Scale-dependent Continua for Complex Materials: A Generalized Voigt Approach Using the Virtual Power Equivalence, in: P. Trovalusci (Ed.), Materials with Internal Structure, Springer International Publishing, 2016, pp. $109-131$.

[8] I. A. Kunin, The Theory of Elastic Media with Microstructure and the Theory of Dislocations, in: E. Kröner (Ed.), Mechanics of Generalized Continua, Springer Berlin Heidelberg, 1968, pp. 321-329.

[9] I. Kunin, Elastic Media with Microstructure: One-dimensional models, Elastic Media with Microstructure, Springer-Verlag, 1982.

[10] I. Kunin, Elastic Media with Microstructure II: ThreeDimensional Models, Elastic Media with Microstructure, Springer-Verlag, 1983.

[11] R. D. Mindlin, Micro-structure in linear elasticity, Arch. Ration. Mech. An. 16 (1) (1964) 51-78.

[12] I. Kunin, On foundations of the theory of elastic media with microstructure, Int. J. Eng. Sci. 22 (8) (1984) 969 - 978.

[13] G. Capriz, Continua with Microstructure, Springer Tracts in Natural Philosophy, Springer-Verlag, 1989.

[14] G. Maugin, Material Inhomogeneities in Elasticity, Applied Mathematics, Taylor \& Francis, 1993.

[15] A. Eringen, Microcontinuum Field Theory, Springer, 1999.

[16] M. Gurtin, Configurational Forces as Basis Concept of Continuum Physics, Springer-Verlag, 1999.

[17] P. Trovalusci, Molecular Approaches for Multifield Continua: Origins and Current Developments, in: T. Sadowski, P. Trovalusci (Eds.), Multiscale Modeling of Complex Materials: Phenomenological, Theoretical and Computational Aspects, Springer Vienna, 2014, pp. 211-278.

[18] E. Cosserat, F. Cosserat, Théorie des Corps Déformables, Herman et fils, Paris, 1909.

[19] W. Nowacki, Theory of Asymmetric Elasticity, Elsevier Science \& Technology, 1986.

[20] H. Altenbach, V. Eremeyev, Generalized Continua - from the Theory to Engineering Applications, CISM International Centre for Mechanical Sciences, Springer Vienna, 2012.

[21] H. Altenbach, S. Forest, Generalized Continua as Models for Classical and Advanced Materials, Advanced Structured Materials, Springer International Publishing, 2016.

[22] R. Masiani, P. Trovalusci, Cosserat and Cauchy materials as continuum models of brick masonry, Meccanica 31 (1996) 421432.

[23] S. Forest, K. Sab, Cosserat overall modeling of heterogeneous materials, Mech. Res. Commun. 25 (1998) $449-454$.

[24] S. Forest, R. Dendievel, G. Canova, Estimating the overall properties of heterogeneous Cosserat materials, Model. Simul. Mater. Sc. 7 (1999) 829-840.

[25] I. Jasiuk, M. Ostoja-Starzewski, From Lattices and Composites to Micropolar Continua, in: V. M. Harik, L.-S. Luo (Eds.), Micromechanics and Nanoscale Effects: MEMS, Multi-Scale Matrials and Micro-Flows, Springer Netherlands, 2004, pp. 175212.

[26] D. Bigoni, W. J. Drugan, Analytical derivation of Cosserat moduli via homogenization of heterogeneous elastic materials, J. Appl. Mech. 74 (2006) 741-753.

[27] J. Li, M. Ostoja-Starzewski, Micropolar continuum mechanics of fractal media, Int. J. Eng. Sci. 49 (2011) $1302-1310$.

[28] A. Beveridge, M. Wheel, D. Nash, The micropolar elastic behaviour of model macroscopically heterogeneous materials, Int. J. Solids Struct. 50 (2013) $246-255$

[29] V. A. Eremeyev, W. Pietraszkiewicz, Material symmetry group and constitutive equations of micropolar anisotropic elastic solids, Math. Mech. Solids 21 (2016) 210-221.

[30] E. Atroshchenko, J. S. Hale, J. Videla, S. Potapenko, S. Bordas, Micro-structured materials: inhomogeneities and imperfect interfaces in plane micropolar elasticity, a boundary element approach, Eng. Anal. Bound. Elem. 83 (2017) 195-203.

[31] M. Godio, I. Stefanou, K. Sab, J. Sulem, S. Sakji, A limit analysis approach based on Cosserat continuum for the evaluation of 
the in-plane strength of discrete media: Application to masonry, Eur. J. Mech.A/Solid 66 (2017) 168 - 192.

[32] V. A. Eremeyev, B. L. Sharma, Anti-plane surface waves in media with surface structure: Discrete vs. continuum model, Int. J. Eng. Sci. 143 (2019) $33-38$.

[33] N. Fantuzzi, P. Trovalusci, S. Dharasura, Mechanical behavior of anisotropic composite materials as micropolar continua, Frontiers in Materials 6 (2019) 59.

[34] N. Fantuzzi, P. Trovalusci, R. Luciano, Material symmetries in homogenized hexagonal-shaped composites as Cosserat continua, Symmetry 12 (2020) 441.

[35] Z. He, H. Zhu, X. Wang, S. Ma, Experimental investigation on scale effect of mechanical properties of heterogeneous micropolar medium materials, Compos. Struct. 251 (2020) 112667.

[36] A. Eringen, D. Edelen, On nonlocal elasticity, Int. J. Eng. Sci. 10 (3) (1972) $233-248$.

37] A. Eringen, Nonlocal Continuum Field Theories, SpringerVerlag, 2002.

[38] A. A. Pisano, A. Sofi, P. Fuschi, Finite element solutions for nonhomogeneous nonlocal elastic problems, Mech. Res. Commun. 36 (2009) $755-761$

[39] P. Fuschi, A. A. Pisano, D. De Domenico, Plane stress problems in nonlocal elasticity: finite element solutions with a straindifference-based formulation, J. Math. Anal. Appl. 431 (2015) $714-736$

[40] H. Zhang, C. Wang, N. Challamel, Small length scale coefficient for Eringen's and lattice-based continualized nonlocal circular arches in buckling and vibration, Compos. Struct. 165 (2017) $148-159$

[41] M. Tuna, M. Kirca, Bending, buckling and free vibration analysis of Euler-bernoulli nanobeams using Eringen's nonlocal integral model via finite element method, Compos. Struct. 179 (2017) $269-284$

[42] M. Tuna, M. Kirca, P. Trovalusci, Deformation of atomic models and their equivalent continuum counterparts using Eringen's two-phase local/nonlocal model, Mech. Res. Commun. 97 (2019) $26-32$.

[43] R. Abdollahi, B. Boroomand, On using mesh-based and meshfree methods in problems defined by Eringen's non-local integral model: issues and remedies, Meccanica 54 (11) (2019) 18011822 .

44] P. Fuschi, A. A. Pisano, C. Polizzotto, Size effects of smallscale beams in bending addressed with a strain-difference based nonlocal elasticity theory, Int. J. Mech. Sci. 151 (2019) 661 671.

[45] M. Eltaher, F. Omar, W. Abdalla, E. Gad, Bending and vibrational behaviors of piezoelectric nonlocal nanobeam including surface elasticity, Wave. Random Complex 29 (2019) 264-280.

[46] M. Fakher, S. Rahmanian, S. Hosseini-Hashemi, On the carbon nanotube mass nanosensor by integral form of nonlocal elasticity, Int. J. Mech. Sci. 150 (2019) 445 - 457.

[47] G. Guclu, R. Artan, Large elastic deflections of bars based on nonlocal elasticity, ZAMM - Z. Angew. Math. Me. (2020) e201900108.

[48] U. Eroglu, Perturbation approach to Eringen's local/non-local constitutive equation with applications to 1-d structures, Meccanica 55 (2020) 1119 - 1134.

[49] A. Naderi, S. Behdad, M. Fakher, S. Hosseini-Hashemi, Vibration analysis of mass nanosensors with considering the axialflexural coupling based on the two-phase local/nonlocal elasticity, Mech. Syst. Signal Pr. 145 (2020) 106931.

[50] P. Phung-Van, A. Ferreira, C. H. Thai, Computational optimization for porosity-dependent isogeometric analysis of functionally graded sandwich nanoplates, Compos. Struct. 239 (2020) 112029.

[51] P. Trovalusci, A. Pau, Derivation of microstructured continua from lattice systems via principle of virtual works: the case of masonry-like materials as micropolar, second gradient and classical continua, Acta Mech. 225 (2014) 157-177.

[52] N. Challamel, C. Wang, I. Elishakoff, Nonlocal or gradient elasticity macroscopic models: A question of concentrated or dis- tributed microstructure, Mech. Res. Commun. 71 (2016) 25 31

[53] V. A. Eremeyev, G. Rosi, S. Naili, Comparison of anti-plane surface waves in strain-gradient materials and materials with surface stresses, Math. Mech. Solids 24 (2019) 2526-2535.

[54] M. Tuna, P. Trovalusci, Scale dependent continuum approaches for discontinuous assemblies: 'explicit' and 'implicit' non-local models, Mech. Res. Commun. 103 (2020) 103461.

[55] M. Tuna, L. Leonetti, P. Trovalusci, M. Kirca, 'Explicit' and 'implicit' non-local continuous descriptions for a circular plate with an inclusion in tensions, Meccanica 55 (2020) 927-944.

[56] M. Tuna, L. Leonetti, P. Trovalusci, M. Kirca, 'Explicit' and 'Implicit' Non-local Continuum Descriptions: Plate with Circular Hole, in: E. Ghavanloo, S. A. Fazelzadeh, F. M. de Sciarra (Eds.), Size-dependent Continuum Mechanics Approaches: Theory and Applications (Accepted), 2020.

[57] P. Kaloni, T. Ariman, Stress concentration effects in micropolar elasticity, Z. Angew. Math. Phys. 18 (1) (1967) 136-141.

[58] B. S. Kim, A. C. Eringen, Stress distribution around an elliptic hole in an infinite domain, Letter Appl. Eng. Sci. 1 (1973) 381390.

[59] A. Basu, A note on stress concentration around an elliptic hole in micropolar elasticity, J. Aust. Math. Soc. B 19 (1976) 289-293.

[60] S. Nakamura, R. Lakes, Finite element analysis of stress concentration around a blunt crack in a Cosserat elastic solid, Comput. Method Appl. M. 66 (1988) 257 - 266.

[61] I. Jasiuk, M. Ostoja-Starzewski, Planar Cosserat elasticity of materials with holes and intrusions, Appl. Mech. Rev. 48 (1995) S11-S18.

[62] F. Y. Huang, K. Z. Liang, Boundary element analysis of stress concentration in micropolar elastic plate, Int. J. Numer. Meth. Eng. 40 (1997) 1611-1622.

[63] F. Tornabene, N. Fantuzzi, M. Bacciocchi, Mechanical behaviour of composite Cosserat solids in elastic problems with holes and discontinuities, Compos. Struct. 179 (2017) $468-481$.

[64] C. E. Inglis, Stresses in a plate due to the presence of cracks and sharp corners, Trans. Inst. Nav. Arch. 55 (1913) $219-241$.

[65] A. A. Pisano, P. Fuschi, Structural symmetry and boundary conditions for nonlocal symmetrical problems, Meccanica 53 (3) (2018) 629-638

[66] C. Polizzotto, Nonlocal elasticity and related variational principles, Int. J. Solids Struct. 38 (2001) 7359-7380.

[67] R. A. Toupin, A note on stress concentration around an elliptic hole in micropolar elasticity, Arc. Ration. Mech. An. 11 (1962) 385-414.

[68] M. Sokolowski, Theory of Couple-stresses in Bodies with Constrained Rotations, Course and Lectures CISM, SpringerVerlag, 1972.

[69] P. A. Gourgiotis, D. Bigoni, Stress channelling in extreme couple-stress materials part I: Strong ellipticity, wave propagation, ellipticity, and discontinuity relations, J. Mech. Phys. Solids 88 (2016) $150-168$.

[70] R. Lakes, Experimental Methods for Study of Cosserat Elastic Solids and Other Generalized Continua, in: H. Mühlhaus (Ed.), Continuum Models for Materials with Micro-structure, John Wiley, New York, 1995, pp. 1-22.

[71] P. Trovalusci, R. Masiani, Material symmetries of micropolar continua equivalent to lattices, Int. J. Solids Struct. 36 (14) (1999) $2091-2108$.

[72] A. C. Eringen, Theory of nonlocal elasticity and some applications, Res Mech. 21 (1987) 313-342.

[73] S. Altan, Uniqueness of initial-boundary value problems in nonlocal elasticity, Int. J. Solids Struct. 25 (11) (1989) $1271-1278$.

74] A. C. Eringen, On differential equations of nonlocal elasticity and solutions of screw dislocaltion and surface waves, J. Appl. Phys. 54 (1983) 4703-4710.

[75] S. Bharath Ram, M. Ramanathan, Shortest path in a multiplyconnected domain having curved boundaries, Comput. Aided Design 45 (3) (2013) 723 - 732 .

76] E. Tadmor, R. Miller, Modeling Materials: Continuum, Atom- 
istic and Multiscale Techniques, Cambridge University Press, 2011.

[77] M. S. Shephard, Linear multipoint constraints applied via transformation as part of a direct stiffness assembly process, Int. J. Numer. Meth. Eng. 20 (11) (1984) 2107-2112.

[78] J. C. G. Orden, R. A. Ortega, A conservative augmented lagrangian algorithm for the dynamics of constrained mechanical systems, Mech. Based Des. Struc. 34 (4) (2006) 449-468.

[79] F. Gonzalez, J. Kovecses, Use of penalty formulations in dynamic simulation and analysis of redundantly constrained multibody systems, Multibody Syst. Dyn. 29 (2013) 57-76.

[80] J. F. Abel, M. S. Shephard, An algorithm for multipoint constraints in finite element analysis, Int. J. Numer. Meth. Eng. 14 (3) (1979) 464-467.

[81] R. Storn, K. Price, Differential Evolution - A simple and efficient heuristic for global optimization over continuous spaces, J. Global Optim. 11 (4) (1997) 341-359.

[82] M. Tuna, M. Kirca, Unification of Eringen's nonlocal parameter through an optimization-based approach, Mec. Adv. Mater. Struc.doi:10.1080/15376494.2019.1601312. 


\section{Declaration of interests}

$\bigotimes$ The authors declare that they have no known competing financial interests or personal relationships that could have appeared to influence the work reported in this paper.

$\square$ The authors declare the following financial interests/personal relationships which may be considered as potential competing interests:

Patrizia TROVALUSCI

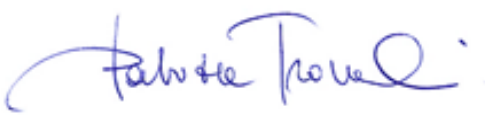

Meral TUNA

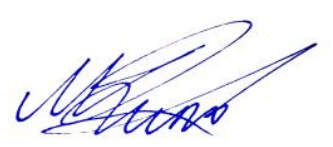

